Appl. Set-Valued Anal. Optim. 3 (2021), No. 2, pp. 239-255

Available online at http://asvao.biemdas.com

https://doi.org/10.23952/asvao.3.2021.2.08

\title{
THE PROJECTION METHOD WITH INERTIAL EXTRAPOLATION FOR SOLVING SPLIT EQUILIBRIUM PROBLEMS IN HILBERT SPACES
}

\author{
FERDINARD UDOCHUKWU OGBUISI
}

Department of Mathematics, University of Nigeria, Nsukka, Nigeria

\begin{abstract}
The purpose of this paper is to introduce an inertial-type iterative algorithm that does not require any knowledge of the operator norm for approximating a solution of the split equilibrium problem with pseudo-monotone bifunctions. Our proposed iterative method is based on the projection and inertial techniques. Under suitable conditions, we present weak and strong convergence results in Hilbert spaces. Keywords. Pseudomotone bifunction; Inertial method; Split equilibrium problem; Projection Method; Hilbert spaces.
\end{abstract}

\section{INTRODUCTION}

Let $C$ be a nonempty closed and convex subset of a real Hilbert space $H$, and let $f: C \times C \rightarrow \mathbb{R}$ be a bifunction. The equilibrium problem is to find $x^{*} \in C$ such that

$$
f\left(x^{*}, x\right) \geq 0, \forall x \in C .
$$

We denote the set of solutions of problem (1.1) by $E P(f, C)$ in this paper, that is,

$$
E P(f, C)=\left\{x^{*} \in C: f\left(x^{*}, x\right) \geq 0, \forall x \in C\right\} .
$$

Equilibrium problems are fundamental in a broad range of mathematical and applied sciences and a lot of problems in structural analysis, machine learning, management sciences and economics, etc; see, e.g., $[1,2,3,4,5]$. The theoretical and algorithmic foundations as well as the applications of the equilibrium problem have been extensively studied in the literature and are attracting intensive attention; see, e.g., $[6,7,8,9,10]$. The equilibrium problem [11] has a lot of applications in several very important mathematical problems such as optimisation problem, saddle points problem, Nash equilibria problem in noncooperative games, convex differentiable optimisation problem, variational operator inequalities problem, complementarity problems and variational inequalities with multivalued mappings.

Let $C$ and $Q$ be nonempty, closed and convex subsets of real Hilbert spaces $H_{1}$ and $H_{2}$, respectively. Let $f_{1}: C \times C \rightarrow \mathbb{R}$ and $f_{2}: Q \times Q \rightarrow \mathbb{R}$ be bifunctions and let $A: H_{1} \rightarrow H_{2}$ be a bounded linear operator. Then the split equilibrium problem (SEP) is to find $x^{*} \in C$ such that

$$
f_{1}\left(x^{*}, x\right) \geq 0, \forall x \in C,
$$

E-mail address: ferdinard.ogbuisi@unn.edu.ng.

Received May 30, 2020; Accepted July 31, 2020.

(C)2021 Applied Set-Valued Analysis and Optimization 
and $y^{*}=A x^{*} \in Q$ is such that

$$
f_{2}\left(y^{*}, y\right) \geq 0, \forall y \in Q
$$

We denote the solution set of (1.2)-(1.3) by $\Omega=\left\{x^{*} \in E P\left(f_{1}, C\right): A x^{*} \in E P\left(f_{2}, Q\right)\right\}$.

Recently, the SEP has received considerable attention; see, e.g., $[12,13,18,14,15]$ and the references therein. The proximal type algorithm which involves using the resolvent of a monotone bifunction have featured prominently in the approximations of solution to SEPs (see, e.g., $[16,17])$ and is mostly applicable when the equilibrium bifunctions are monotone. Hieu [18] introduced an iterative method, which combines the extragradient-like method with the proximal method for the approximation of solutions of SEPs in Hilbert spaces. The extragradient-like methods are more applicable because they can be used to solve EPs with pseudomonotone bifunctions and the subproblems can be numerically solved more easily than the subproblems in the proximal method; see, e.g., [18, 19, 20, 21, 22]. However, the problems of solving optimization subproblems and of finding shrinking projections [18] can be costly if bifunctions and feasible sets have complex structures.

In 2016, Hieu et al. [22] proposed an iterative algorithm called the projected subgradientproximal algorithm, which combines the diagonal subgradient method, the projection method and the proximal method for solving SEPs in Hilbert spaces. The advantage of this algorithm over the iterative algorithm proposed in [18] lies in its simple nature and more importantly in the fact that the metric projection, in general, is easier to compute than optimization problems on the same feasible set. But this algorithm is not without its own set back as the step-size in the second projection is chosen in a real interval which depends on the norm of operator. The problem of finding or at least an estimate of the norm of an operator, in general, is not an easy task in Hilbert spaces. To overcome this drawback, Hieu et al. in [22] come out with a modified version of the first algorithm where the second projection is performed on feasible set while the first projection is on a specifically constructed half-space, and thus, it is explicit. Moreover, it involves a way of selecting an adaptive step-size in the second projection such that the prior knowledge of operator norm is not required.

The two algorithms proposed in [22] involve the use of the proximal point method [23] (i.e., using the resolvent mapping of bifunction $f_{2}$ ) and as it is well known, the computation of the value of resolvent mapping in general is not easy. Thus, this raises the natural question of whether it is possible to construct an algorithm for solving SEP which only uses the projection method. Hieu [24] gave an affirmative answer to this question by proposing an iterative algorithm that is designed only by the use of the projection method for solving SEPs.

Precisely, the iterative algorithm proposed in [24] is as follows:

Algorithm 1.1. Initialization: Choose $x_{0} \in C$ and four non-negative parameter sequences $\left\{\rho_{n}\right\},\left\{\beta_{n}\right\},\left\{\varepsilon_{n}\right\}$ and $\left\{\mu_{n}\right\}$ satisfying the following conditions.

c1. $\rho_{n} \geq \rho>0, \beta_{n}>0, \varepsilon_{n} \geq 0$.

c2. $\sum_{n=1}^{\infty} \frac{\beta_{n}}{\rho_{n}}=+\infty, \sum_{n=1}^{\infty} \frac{\beta_{n} \varepsilon_{n}}{\rho_{n}}<+\infty, \sum_{n=1}^{\infty} \beta_{n}^{2}<+\infty$.

c3. $0<a \leq \mu_{n} \leq \frac{1}{\|A\|^{2}}$. 
Assume that $x_{n} \in C$ is known, and calculate $x_{n+1}$ by the following steps:

Step 1: Select $w_{n} \in \partial_{\varepsilon_{n}} f_{2}\left(u_{n},.\right)\left(u_{n}\right)$ where $u_{n}=P_{Q}\left(A x_{n}\right)$, and compute

$$
\gamma_{n}=\frac{\beta_{n}}{\max \left\{\rho_{n},\left\|w_{n}\right\|\right\}}, y_{n}=P_{Q}\left(u_{n}-\gamma_{n} w_{n}\right) .
$$

Step 2: Compute $z_{n}=P_{C}\left(x_{n}+\mu_{n} A^{*}\left(y_{n}-A x_{n}\right)\right)$.

Step 3: Select $g_{n} \in \partial_{\varepsilon_{n}} f_{1}\left(z_{n},.\right)\left(z_{n}\right)$ and compute

$$
\alpha_{n}=\frac{\beta_{n}}{\max \left\{\rho_{n},\left\|g_{n}\right\|\right\}}, x_{n+1}=P_{C}\left(z_{n}-\alpha_{n} g_{n}\right) .
$$

Set $n:+n+1$ and go to Step 1 .

Obviously, when the operator norm is difficult to compute or approximate, the iterative algorithm proposed in [24] suffers a set back since the step-size $\mu_{n}$ depends on $\|A\|$. In iterative methods, the need to speed up the convergence of iterative algorithms is a very natural problem because algorithms with faster rate of convergence are always more effective and less costly. In order to obtain the iterative algorithms with better rate of convergence than the previous ones in the literature, many authors have studied inertial type algorithms such as, the inertial proximal point algorithm $[2,25]$ (which is based upon a discrete version of a second order dissipative dynamical system [3, 4]), the inertial forward-backward splitting methods [26, 27, 28], the inertial Douglas-Rachford splitting method [29], the inertial ADMM [30], and the inertial forwardbackward-forward method [31]. These results analysed the convergence properties of inertial extrapolation type algorithms and demonstrated their improved performance numerically on some imaging and data analysis problems.

Recently, Tian and Tong [32] proposed an inertial type algorithm for approximating a common solution of a variational inequality problem and a fixed point problem as follows:

Algorithm 1.2. Step 1: Choose $x_{0}, x_{1} \in H, \mu \in(0,1), \lambda_{1}>0$.

Step 2: Set $w_{n}=x_{n}+\theta_{n}\left(x_{n}-x_{n-1}\right)$, and compute

$$
y_{n}=P_{C}\left(w_{n}-\lambda_{n} A w_{n}\right) .
$$

Step 3: Compute

$$
z_{n}=P_{T_{n}}\left(w_{n}-\lambda_{n} A y_{n}\right),
$$

where $T_{n}=\left\{x \in H \mid\left\langle w_{n}-\lambda_{n} A w_{n}-y_{n}, x-y_{n}\right\rangle \leq 0\right\}$.

Step 4: Compute

$$
x_{n+1}=\left(1-\beta_{n}\right) w_{n}+\beta_{n} T z_{n}
$$

and

$$
\lambda_{n+1}=\left\{\begin{array}{l}
\min \left\{\frac{\mu\left\|x_{n}-y_{n}\right\|^{2}}{\left\|A x_{n}-A y_{n}\right\|^{2}}, \lambda_{n}\right\}, \text { if }\left\|A x_{n}-A y_{n}\right\| \neq 0 \\
\lambda_{n}, \\
\text { Otherwise. }
\end{array}\right.
$$

If $w_{n}=y_{n}=x_{n+1}$, then $w_{n} \in F(T) \cap V I(C, A)$, where $F(T)$ stands for the fixed point set of $T$ and $\operatorname{VI}(C, A)$ stands for the set of solutions of the variational inequality problem. Set $n:=n+1$ and return to Step 2 . 
In the work of Tian and Tong [32], one of the conditions imposed on the inertial term is $0 \leq \theta_{n} \leq \theta \leq \frac{1}{4}$ which is very restrictive on the inertial term. Long et al. [33] proposed an inertial iterative algorithm with a very mild condition on the inertial term for solving split variational inclusion problems as follows.

Algorithm 1.3. Assume that the sequence $\left\{\theta_{n}\right\}$ is non-decreasing such that $0 \leq \theta_{n} \leq \theta<1$. Let $\beta>0$ and $\left\{\lambda_{n}\right\}$ be a sequence of real numbers such that $0<a \leq \lambda_{n} \leq b<\frac{1}{L}$, where $L:=\|A\|^{2}$ and the sequence $\left\{\alpha_{n}\right\}$ is non-decreasing such that $\alpha<\alpha_{n} \leq \frac{1}{1+\theta+\sigma}, \alpha>0, \sigma>0$, Then $\left\{x_{n}\right\}$ in $H_{1}$ is generated as follows:

$$
\left\{\begin{array}{l}
x_{0}, x_{1} \in H_{1} \\
w_{n}=x_{n}+\theta_{n}\left(x_{n}-x_{n-1}\right) \\
y_{n}=J_{\beta}^{B_{1}}\left(I-\lambda_{n} A^{*}\left(I-J_{\beta}^{B_{2}}\right) A\right) w_{n} \\
x_{n+1}=\left(1-\alpha_{n}\right) x_{n}+\alpha_{n} y_{n} .
\end{array}\right.
$$

Again, we can see that the result of Long et al. [33] requires a prior knowledge of the operator norm which may be problematic, since the operator norm in general is not easy to compute or estimate.

Inspired by the recent interest on the inertial-type algorithms, and the results in Hieu [24], Tian and Tong [32] and Long et al. [33], we propose an exclusively projection algorithm which involves an inertial extrapolation step as well as a self adaptive step size which does not require any knowledge of the operator norm for solving SEPs in real Hilbert spaces. Under some mild assumptions on the associated bifunctions, the control sequences and the inertial factor, we establish weak and strong convergence results using our proposed method.

The clear advantages of our result over the result of Hieu [24] is that our algorithm involves an inertial extrapolation term which is not present in the algorithm of Hieu [24], whereas, the convergence analysis of the algorithms proposed in Hieu [24] and Long et al. [33] require the prior knowledge of the operator norm. Our proposed algorithm is independent of the operator norm. Furthermore, our iterative algorithm utilizes the type of conditions on the inertial term as in the work of Long et al. which is less restrictive than the condition imposed on inertial term in the work of Tian and Tong [32]. Moreover our result partially extends the work of Tian and Tong [32] from variational inequality problems to split equilibrium problems.

\section{PRELIMINARIES}

Let $C$ be a nonempty closed and convex subset of a real Hilbert space $H$. The metric projection $P_{C}: H \rightarrow C$ is defined by

$$
P_{C}:=\arg \min \{\|y-x\|: y \in C\} .
$$

Since $C$ is nonempty, closed and convex, $P_{C}(x)$ exists and is unique.

The following two lemmas are trivial.

Lemma 2.1. The metric projection $P_{C}$ has the following properties.

i. $\left.\left\langle P_{C}(x)-P_{C}(y), x-y\right\rangle \geq \| P_{C}(x)-P_{(} y\right) \|^{2} ; \forall x, y \in H$,

ii. $\left\|x-P_{C}(y)\right\|^{2}+\left\|P_{C}(y)-y\right\|^{2} \leq\|x-y\|^{2} ; \forall x \in C, y \in H$,

iii. $z=P_{C}(x) \Leftrightarrow\langle x-z, y-z\rangle \leq 0 ; \forall y \in C$. 
Lemma 2.2. For each $x, y \in H$ and $\alpha \in \mathbb{R}$, we have

$$
\begin{gathered}
\|x+y\|^{2} \leq\|x\|^{2}+2\langle y, x+y\rangle . \\
\|\alpha x+(1-\alpha) y\|^{2}=\alpha\|x\|^{2}+(1-\alpha)\|y\|^{2}-\alpha(1-\alpha)\|x-y\|^{2} .
\end{gathered}
$$

Lemma 2.3. [2] Let $\left\{\varphi_{n}\right\},\left\{\delta_{n}\right\}$ and $\left\{\alpha_{n}\right\}$ be sequences in $[0,+\infty)$ such that

$$
\varphi_{n+1} \leq \varphi_{n}+\alpha_{n}\left(\varphi_{n}-\varphi_{n-1}\right)+\delta_{n} \forall n \geq 1, \sum_{n=1}^{\infty} \delta_{n}<+\infty
$$

and assume that there exists a real number $\alpha$ with $0 \leq \alpha_{n} \leq \alpha<1$ for all $n \in \mathbb{N}$. Then the following hold:

i. $\sum_{n=1}^{\infty}\left[\varphi_{n}-\varphi_{n-1}\right]_{+}<\infty$, where $[t]_{+}:=\max \{t, 0\}$;

ii. there exists $\varphi^{*} \in[0,+\infty)$ such that $\lim _{n \rightarrow+\infty} \varphi_{n}=\varphi^{*}$.

Lemma 2.4. [34] Let $\left\{a_{n}\right\}$ and $\left\{b_{n}\right\}$ be two sequences of positive real numbers such that

$$
a_{n+1} \leq a_{n}+b_{n}, \forall n \geq 1
$$

with $\sum_{n \geq 1} b_{n}<+\infty$. Then the sequence $\left\{a_{n}\right\}$ is convergent.

Lemma 2.5. Let $C$ be a nonempty subset of $H$ and $\left\{x_{n}\right\}$ be a sequence in $H$ such that the following two conditions hold:

(i) for every $x \in C, \lim _{n \rightarrow \infty}\left\|x_{n}-x\right\|$ exists;

(ii) every sequential weak cluster point of $\left\{x_{n}\right\}$ is in $C$.

Then $\left\{x_{n}\right\}$ converges weakly to a point in $C$.

Lemma 2.6. [35] Assume $\left\{a_{n}\right\}$ is a sequence of nonnegative real numbers such that

$$
a_{n+1} \leq\left(1-\gamma_{n}\right) a_{n}+\gamma_{n} \delta_{n}, n \geq 0,
$$

where $\left\{\gamma_{n}\right\}$ is a sequence in $(0,1)$ and $\left\{\delta_{n}\right\}$ is a sequence in $\mathbb{R}$ such that

(i) $\sum_{n=0}^{\infty} \gamma_{n}=\infty$

(ii) $\limsup _{n \rightarrow \infty} \delta_{n} \leq 0$ or $\sum_{n=0}^{\infty}\left|\delta_{n} \gamma_{n}\right|<\infty$,

Then $\lim _{n \rightarrow \infty} a_{n}=0$.

Definition 2.1. A bifunction $f: C \times C \rightarrow \mathbb{R}$ is said to be

i. strongly monotone on $C$ if there exists a constant $\gamma>0$ such that

$$
f(x, y)+f(y, x) \leq-\gamma\|x-y\|^{2} ; \forall x, y \in C ;
$$

ii. monotone on $C$ if

$$
f(x, y)+f(y, x) \leq 0 ; \forall x, y \in C
$$

iii. pseudomonotone on $C$ if

$$
f(x, y) \geq 0 \Rightarrow f(y, x) \leq 0 ; \forall x, y \in C ;
$$

iv. strongly pseudomonotone on $C$ if there exists a constant $\gamma>0$ such that

$$
f(x, y) \geq 0 \Rightarrow f(y, x) \leq-\gamma\|x-y\|^{2} ; \forall x, y \in C .
$$


From the above definitions, it is clear that the following implications hold, $(i) \Rightarrow(i i) \Rightarrow(i i i)$ and $(i) \Rightarrow(i v) \Rightarrow(i i i)$, but the converses in general are not true.

Recall that a function $\varphi: C \rightarrow \mathbb{R}$ is said to be convex on $C$ if, for all $x, y \in C$ and $t \in[0,1]$,

$$
\varphi(t x+(1-t) y) \leq t \varphi(x)+(1-t) \varphi(y) .
$$

The subdifferential of $\varphi$ at $x \in C$ is defined by

$$
\partial \varphi(x)=\{w \in H: \varphi(y)-\varphi(x) \geq\langle w, y-x\rangle ; \forall y \in C\} .
$$

An enlargement of the subdifferential is the $\varepsilon$-subdifferential. The $\varepsilon$-subdifferential of $\varphi$ at $x \in C$ is defined by

$$
\partial_{\varepsilon} \varphi(x)=\{w \in H: \varphi(y)-\varphi(x)+\varepsilon \geq\langle w, y-x\rangle ; \forall y \in C\} .
$$

It is clear that the 0 -subdifferential coincides with the subdifferential. Let $f: C \times C \rightarrow \mathbb{R}$ be a bifunction. Throughout this paper, $\partial_{\varepsilon} f(x ; \cdot)(x)$ is called the $\varepsilon$-diagonal subdifferential of $f$ at $x \in C$.

\section{MAin RESUlTS}

In this section, we give our iterative algorithm and the convergence result. We shall assume that the bifunction $f_{1}: C \times C \rightarrow \mathbb{R}$ satisfies the following conditions:

A1. $f_{1}$ is pseudomonotone and $f_{1}(x, x)=0, \forall x \in C$;

A2. $f_{1}(x, \cdot)$ is convex and lower semicontinuous on $C$ and $f_{1}(\cdot, y)$ is weakly upper semicontinuous on $C$;

A3. the $\varepsilon$-diagonal subdifferential of $f_{1}$ is bounded on each subset of $C$;

A4. $f_{1}$ satisfies the following paramonotone condition

$$
x \in E P\left(f_{1}, C\right) ; y \in C ; f_{1}(y, x)=0 \Rightarrow y \in E P\left(f_{1}, C\right) .
$$

We also require that $f_{2}$ satisfies conditions $A 1-A 4$ on $Q$. Furthermore, it has been noted that under conditions $A 1$ and $A 2$, the two sets $E P\left(f_{1}, C\right)$ and $E P\left(f_{2}, Q\right)$ are closed and convex. Thus, since $A$ is linear, the solution set $\Omega$ of problem (SEP) is also closed and convex.

Algorithm 3.1. For initial $x_{0}, x_{1} \in C, \lambda_{1}>0, \mu \in(0,1)$ and non-negative parameter sequences $\left\{\rho_{n}\right\},\left\{\beta_{n}\right\},\left\{\tau_{n}\right\},\left\{\theta_{n}\right\}$ and $\left\{\varepsilon_{n}\right\}$ satisfying the following conditions,

1. $\rho_{n} \geq \rho>0, \beta_{n}>0, \varepsilon_{n}>0$,

2. $\sum_{n=1}^{\infty} \frac{\beta_{n} \varepsilon_{n}}{\rho_{n}}<+\infty, \sum_{n=1}^{\infty} \beta_{n}^{2}<+\infty$,

3. $\left\{\tau_{n}\right\}$ and $\left\{\theta_{n}\right\}$ are nondecreasing sequences,

4. $0 \leq \theta_{n} \leq 1, \tau<\tau_{n} \leq \frac{1}{2+\sigma}, \tau>0, \sigma>0$,

calculate $x_{n+1}$ as follows:

Step 1. Set $w_{n}=x_{n}+\theta_{n}\left(x_{n}-x_{n-1}\right)$, and select $v_{n} \in \partial_{\varepsilon_{n}} f_{2}\left(u_{n}, \cdot\right)\left(u_{n}\right)$, where $u_{n}=P_{Q}\left(A w_{n}\right)$, and compute

$\gamma_{n}=\frac{\beta_{n}}{\max \left\{\rho_{n},\left\|v_{n}\right\|\right\}}, y_{n}=P_{Q_{n}}\left(u_{n}-\gamma_{n} v_{n}\right)$, where $Q_{n}$ is the set defined by

$$
Q_{n}=\left\{y \in H_{2}:\left\langle y-u_{n}, A w_{n}-u_{n}\right\rangle \leq 0\right\} .
$$


Step 2. Compute $z_{n}=P_{C}\left(w_{n}+\lambda_{n} A^{*}\left(y_{n}-A w_{n}\right)\right)$ and

$$
\lambda_{n+1}=\left\{\begin{array}{l}
\min \left\{\frac{\mu\left\|y_{n}-A w_{n}\right\|^{2}}{\left\|A^{*}\left(y_{n}-A w_{n}\right)\right\|^{2}}, \lambda_{n}\right\}, \text { if }\left\|A^{*}\left(y_{n}-A w_{n}\right)\right\| \neq 0, \\
\lambda_{n}, \\
\text { Otherwise. }
\end{array}\right.
$$

Step 3. Select $g_{n} \in \partial_{\varepsilon_{n}} f_{1}\left(z_{n}, \cdot\right)\left(z_{n}\right)$ and compute

$$
\alpha_{n}=\frac{\beta_{n}}{\max \left\{\rho_{n},\left\|g_{n}\right\|\right\}}, x_{n+1}=\left(1-\tau_{n}\right) x_{n}+\tau_{n} P_{C_{n}}\left(z_{n}-\alpha_{n} g_{n}\right) .
$$

The set $C_{n}$ is defined by

$$
C_{n}=\left\{x \in H_{1}:\left\langle x-z_{n}, w_{n}+\lambda_{n} A^{*}\left(y_{n}-A w_{n}\right)-z_{n}\right\rangle \leq 0\right\} .
$$

Remark 3.1. Clearly, the half spaces $C_{n}$ and $Q_{n}$ contain $C$ and $Q$, respectively.

Theorem 3.1. Let $\left\{x_{n}\right\}$ be the sequence generated by Algorithm 3.1 and suppose that $\Omega \neq \emptyset$. Then $\left\{x_{n}\right\}$ converges weakly to some $x^{+} \in \Omega$.

Proof. Clearly, $\left\{\lambda_{n}\right\}$ is a monotonically decreasing sequence. Moreover, if $\left\|A^{*}\left(y_{n}-A w_{n}\right)\right\| \neq 0$, then

$$
\frac{\mu\left\|y_{n}-A w_{n}\right\|^{2}}{\left\|A^{*}\left(y_{n}-A w_{n}\right)\right\|^{2}} \geq \frac{\mu\left\|y_{n}-A w_{n}\right\|^{2}}{\left.\|A\|\right|^{2}\left\|y_{n}-A w_{n}\right\|^{2}}=\frac{\mu}{\|A\|^{2}} .
$$

Therefore, $\left\{\lambda_{n}\right\}$ is monotonically decreasing and bounded below, thus it converges.

Since $x^{*} \in C$, and $C \subset C_{n}$, then $x^{*} \in C_{n}$. Therefore, letting $h_{n}=P_{C_{n}}\left(z_{n}-\alpha_{n} g_{n}\right)$, we conclude from Lemma 2.1(iii) that

$$
\left\langle x^{*}-h_{n}, z_{n}-\alpha_{n} g_{n}-h_{n}\right\rangle \leq 0
$$

From (3.2), we obtain

$$
\begin{aligned}
\left\langle x^{*}-h_{n}, z_{n}-h_{n}\right\rangle & \leq \alpha_{n}\left\langle g_{n}, x^{*}-h_{n}\right\rangle \\
& =\alpha_{n}\left\langle g_{n}, x^{*}-z_{n}\right\rangle+\alpha_{n}\left\langle g_{n}, z_{n}-h_{n}\right\rangle \\
& \leq \alpha_{n}\left\langle g_{n}, x^{*}-z_{n}\right\rangle+\alpha_{n}|| g_{n}|||| z_{n}-h_{n} \| .
\end{aligned}
$$

Also, since $z_{n} \in C_{n}$, we have from Lemma 2.1(iii) that $\left\langle z_{n}-h_{n}, z_{n}-\alpha_{n} g_{n}-h_{n}\right\rangle \leq 0$, which implies

$$
\left\|z_{n}-h_{n}\right\|^{2} \leq \alpha_{n}\left\langle z_{n}-h_{n}, g_{n}\right\rangle \leq \alpha_{n}\left\|g_{n}\right\|\left\|z_{n}-h_{n}\right\|
$$

Hence,

$$
\left\|z_{n}-h_{n}\right\| \leq \alpha_{n}\left\|g_{n}\right\|
$$

Furthermore, from the definition of $\alpha_{n}$, we have

$$
\begin{aligned}
\alpha_{n}|| g_{n}|||| z_{n}-h_{n}|| & \leq\left(\alpha_{n}|| g_{n}||\right)^{2}=\left(\frac{\beta_{n}|| g_{n} \|}{\max \left\{\rho_{n}, \| g_{n}||\right\}}\right)^{2} \\
& =\beta_{n}^{2}\left(\frac{\left\|g_{n}\right\|}{\max \left\{\rho_{n}, \| g_{n}||\right\}}\right)^{2} \leq \beta_{n}^{2} .
\end{aligned}
$$

From assumption A1 and $g_{n} \in \partial_{\varepsilon_{n}} f_{1}\left(z_{n}, \cdot\right)\left(z_{n}\right)$, we have

$$
f_{1}\left(z_{n}, x^{*}\right)+\varepsilon_{n}=f_{1}\left(z_{n}, x^{*}\right)-f_{1}\left(z_{n}, z_{n}\right)+\varepsilon_{n} \geq\left\langle g_{n}, x^{*}-z_{n}\right\rangle .
$$


Again,

$$
\alpha_{n}=\frac{\beta_{n}}{\max \left\{\rho_{n},\left\|g_{n}\right\|\right\}} \leq \frac{\beta_{n}}{\rho_{n}} .
$$

It then follows from (3.3),(3.4),(3.5) and (3.6) that

$$
\left\langle x^{*}-h_{n}, z_{n}-h_{n}\right\rangle \leq \alpha_{n} f_{1}\left(z_{n}, x^{*}\right)+\frac{\beta_{n} \varepsilon_{n}}{\rho_{n}}+\beta_{n}^{2} .
$$

On the other hand,

$$
2\left\langle x^{*}-h_{n}, z_{n}-h_{n}\right\rangle=\left\|x^{*}-h_{n}\right\|^{2}+\left\|z_{n}-h_{n}\right\|^{2}-\left\|z_{n}-x^{*}\right\|^{2} .
$$

Thus, from (3.7) and (3.8), we obtain

$$
\left\|h_{n}-x^{*}\right\|^{2} \leq\left\|z_{n}-x^{*}\right\|^{2}+2 \alpha_{n} f_{1}\left(z_{n}, x^{*}\right)-\left\|z_{n}-h_{n}\right\|^{2}+2 \frac{\beta_{n} \varepsilon_{n}}{\rho_{n}}+2 \beta_{n}^{2} .
$$

By similar arguments as in (3.2)-(3.8), we have

$$
\left\|y_{n}-A x^{*}\right\|^{2} \leq\left\|u_{n}-A x^{*}\right\|^{2}+2 \gamma_{n} f_{2}\left(u_{n}, A x^{*}\right)-\left\|y_{n}-u_{n}\right\|^{2}+\frac{2 \beta_{n} \varepsilon_{n}}{\rho_{n}}+2 \beta_{n}^{2} .
$$

Now,

$$
\begin{aligned}
\left\|x_{n+1}-x^{*}\right\|^{2}= & \left(1-\tau_{n}\right)\left\|x_{n}-x^{*}\right\|^{2}+\tau_{n}\left\|h_{n}-x^{*}\right\|^{2}-\tau_{n}\left(1-\tau_{n}\right)\left\|x_{n}-h_{n}\right\|^{2} \\
\leq & \left(1-\tau_{n}\right)|| x_{n}-x^{*}\left\|^{2}+\tau_{n}\right\| z_{n}-x^{*} \|^{2}+2 \alpha_{n} \tau_{n} f_{1}\left(z_{n}, x^{*}\right) \\
& -\tau_{n}\left\|z_{n}-h_{n}\right\|^{2}+\frac{2 \tau_{n} \beta_{n} \varepsilon_{n}}{\rho_{n}}+2 \beta_{n}^{2} \tau_{n}-\tau_{n}\left(1-\tau_{n}\right)\left\|x_{n}-h_{n}\right\|^{2}
\end{aligned}
$$

But

$$
\begin{aligned}
\left\|z_{n}-x^{*}\right\|^{2} \leq & \left\|w_{n}+\lambda_{n} A^{*}\left(y_{n}-A w_{n}\right)-x^{*}\right\|^{2} \\
= & \left\|w_{n}-x^{*}\right\|^{2}+\lambda_{n}^{2}\left\|A^{*}\left(y_{n}-A w_{n}\right)\right\|^{2} \\
& +2 \lambda_{n}\left\langle A\left(w_{n}-x^{*}\right), y_{n}-A w_{n}\right\rangle .
\end{aligned}
$$

Moreover,

$$
2\left\langle A\left(w_{n}-x^{*}\right), y_{n}-A w_{n}\right\rangle=\left\|y_{n}-A x^{*}\right\|^{2}-\left\|A w_{n}-A x^{*}\right\|^{2}-\left\|y_{n}-A w_{n}\right\|^{2} .
$$

Therefore, from (3.10) and (3.13), we get

$$
\begin{aligned}
2\left\langle A\left(w_{n}-x^{*}\right), y_{n}-A w_{n}\right\rangle \leq & \left\|u_{n}-A x^{*}\right\|^{2}+2 \gamma_{n} f_{2}\left(u_{n}, A x^{*}\right)-\left\|y_{n}-u_{n}\right\|^{2} \\
& +\frac{2 \beta_{n} \varepsilon_{n}}{\rho_{n}}+2 \beta_{n}^{2}-\left\|A w_{n}-A x^{*}\right\|^{2}-\left\|y_{n}-A w_{n}\right\|^{2} .
\end{aligned}
$$

Also, since $P_{Q}$ is firmly nonexpansive, we have

$$
\begin{aligned}
2\left\|u_{n}-A x^{*}\right\|^{2} & =2\left\|P_{Q}\left(A w_{n}\right)-P_{Q}\left(A x^{*}\right)\right\|^{2} \\
& \leq 2\left\langle P_{Q}\left(A w_{n}\right)-P_{Q}\left(A x^{*}\right), A w_{n}-A x^{*}\right\rangle \\
& =2\left\langle u_{n}-A x^{*}, A w_{n}-A x^{*}\right\rangle \\
& =\left\|u_{n}-A x^{*}\right\|^{2}+\left\|A w_{n}-A x^{*}\right\|^{2}-\left\|u_{n}-A w_{n}\right\|^{2}
\end{aligned}
$$

which implies

$$
\left\|u_{n}-A x^{*}\right\|^{2} \leq\left\|A w_{n}-A x^{*}\right\|^{2}-\left\|u_{n}-A w_{n}\right\|^{2}
$$


Combining (3.14) and (3.16), we have

$$
\begin{aligned}
2\left\langle A\left(w_{n}-x^{*}\right), y_{n}-A w_{n}\right\rangle \leq & -\left\|u_{n}-A w_{n}\right\|^{2}-\left\|y_{n}-A w_{n}\right\|^{2} \\
& +2 \gamma_{n} f_{2}\left(u_{n}, A x^{*}\right)+\frac{2 \beta_{n} \varepsilon_{n}}{\rho_{n}}+\beta_{n}^{2} .
\end{aligned}
$$

Substituting for $2\left\langle A\left(w_{n}-x^{*}\right), y_{n}-A w_{n}\right\rangle$ into (3.12), we obtain

$$
\begin{aligned}
\left\|z_{n}-x^{*}\right\|^{2} \leq & \left\|w_{n}-x^{*}\right\|^{2}+\lambda_{n}^{2}\left\|A^{*}\left(y_{n}-A w_{n}\right)\right\|^{2}-\lambda_{n}\left\|u_{n}-A w_{n}\right\|^{2} \\
& -\lambda_{n}\left\|y_{n}-A w_{n}\right\|^{2}+2 \gamma_{n} \lambda_{n} f_{2}\left(u_{n}, A x^{*}\right)+\frac{2 \beta_{n} \lambda_{n} \varepsilon_{n}}{\rho_{n}}+\beta_{n}^{2} \lambda_{n} .
\end{aligned}
$$

Recall that the limit of $\left\{\lambda_{n}\right\}$ exists and $\frac{\lambda_{n}}{\lambda_{n+1}} \geq 1$. Let $\lambda=\lim _{n \rightarrow \infty} \lambda_{n}$. Thus $\lim _{n \rightarrow \infty} \frac{\lambda_{n}}{\lambda_{n+1}}=1$. Also, since $\mu \in(0,1), \frac{1}{\mu}>1$, let $\kappa=\frac{1+\frac{1}{\mu}}{2}$, then $1<\kappa<\frac{1}{\mu}$. Hence, $\exists N \in \mathbb{N}$, such that $\forall n>N, \frac{\lambda_{n}}{\lambda_{n+1}}<\kappa$. Therefore, for all $n>N$, we have $1-\mu \kappa>0$ and

$$
\begin{aligned}
\left\|z_{n}-x^{*}\right\|^{2} \leq & \left\|w_{n}-x^{*}\right\|^{2}+\lambda_{n}^{2} \frac{\mu}{\lambda_{n+1}}\left\|y_{n}-A w_{n}\right\|^{2}-\lambda_{n}\left\|y_{n}-A w_{n}\right\|^{2} \\
& -\lambda_{n}\left\|u_{n}-A w_{n}\right\|^{2}+2 \gamma_{n} \lambda_{n} f_{2}\left(u_{n}, A x^{*}\right)+\frac{2 \beta_{n} \lambda_{n} \varepsilon_{n}}{\rho_{n}}+\beta_{n}^{2} \lambda_{n} \\
\leq & \left\|w_{n}-x^{*}\right\|^{2}-\lambda_{n}(1-\mu \kappa)|| y_{n}-A w_{n}\left\|^{2}-\lambda_{n}\right\| u_{n}-A w_{n} \|^{2} \\
& +2 \gamma_{n} \lambda_{n} f_{2}\left(u_{n}, A x^{*}\right)+\frac{2 \beta_{n} \lambda_{n} \varepsilon_{n}}{\rho_{n}}+\beta_{n}^{2} \lambda_{n} .
\end{aligned}
$$

Thus,

$$
\begin{aligned}
\left\|x_{n+1}-x^{*}\right\|^{2}= & \left(1-\tau_{n}\right)\left\|x_{n}-x^{*}\right\|^{2}+\tau_{n}\left\|w_{n}-x^{*}\right\|^{2}-\tau_{n} \lambda_{n}(1-\mu \kappa)\left\|y_{n}-A w_{n}\right\|^{2} \\
& -\lambda_{n} \tau_{n}\left\|u_{n}-A w_{n}\right\|^{2}+2 \gamma_{n} \tau_{n} \lambda_{n} f_{2}\left(u_{n}, A x^{*}\right)+\frac{2 \tau_{n} \beta_{n} \lambda_{n} \varepsilon_{n}}{\rho_{n}}+\tau_{n} \beta_{n}^{2} \lambda_{n} \\
& +2 \alpha_{n} \tau_{n} f_{1}\left(z_{n}, x^{*}\right)-\tau_{n}\left\|z_{n}-h_{n}\right\|^{2}+\frac{2 \tau_{n} \beta_{n} \varepsilon_{n}}{\rho_{n}}+2 \beta_{n}^{2} \tau_{n} \\
& -\tau_{n}\left(1-\tau_{n}\right)\left\|x_{n}-h_{n}\right\|^{2}
\end{aligned}
$$

Furthermore,

$$
\begin{aligned}
\left\|w_{n}-x^{*}\right\|^{2}= & \left\|\left(1+\theta_{n}\right)\left(x_{n}-x^{*}\right)-\theta_{n}\left(x_{n-1}-x^{*}\right)\right\|^{2} \\
= & \left(1+\theta_{n}\right)\left\|x_{n}-x^{*}\right\|^{2}-\theta_{n}\left\|x_{n-1}-x^{*}\right\|^{2} \\
& +\theta_{n}\left(1+\theta_{n}\right)\left\|x_{n}-x_{n-1}\right\|^{2} .
\end{aligned}
$$


In view of $h_{n}-x_{n}=\frac{1}{\tau_{n}}\left(x_{n+1}-x_{n}\right)$, we conclude from (3.20) and (3.21) that

$$
\begin{aligned}
& \left\|x_{n+1}-x^{*}\right\|^{2} \\
\leq & \left(1-\tau_{n}\right)\left\|x_{n}-x^{*}\right\|^{2}+\tau_{n}\left(1+\theta_{n}\right)\left\|x_{n}-x^{*}\right\|^{2}-\tau_{n} \theta_{n}\left\|x_{n-1}-x^{*}\right\|^{2} \\
& +\tau_{n} \theta_{n}\left(1+\theta_{n}\right)\left\|x_{n}-x_{n-1}\right\|^{2}-\tau_{n} \lambda_{n}(1-\mu \kappa)\left\|y_{n}-A w_{n}\right\|^{2} \\
& -\lambda_{n} \tau_{n}\left\|u_{n}-A w_{n}\right\|^{2}+2 \gamma_{n} \tau_{n} \lambda_{n} f_{2}\left(u_{n}, A x^{*}\right)+\frac{2 \tau_{n} \beta_{n} \lambda_{n} \varepsilon_{n}}{\rho_{n}}+\tau_{n} \beta_{n}^{2} \lambda_{n} \\
& +2 \alpha_{n} \tau_{n} f_{1}\left(z_{n}, x^{*}\right)-\tau_{n}\left\|z_{n}-h_{n}\right\|^{2}+\frac{2 \tau_{n} \beta_{n} \varepsilon_{n}}{\rho_{n}}+2 \beta_{n}^{2} \tau_{n}-\frac{1-\tau_{n}}{\tau_{n}}\left\|x_{n+1}-x_{n}\right\|^{2} \\
\leq & \left(1+\tau_{n} \theta_{n}\right)|| x_{n}-x^{*}\left\|^{2}-\tau_{n} \theta_{n}\right\| x_{n-1}-x^{*}\left\|^{2}+\tau_{n} \theta_{n}\left(1+\theta_{n}\right)\right\| x_{n}-x_{n-1} \|^{2} \\
& -\tau_{n} \lambda_{n}(1-\mu \kappa)\left\|y_{n}-A w_{n}\right\|^{2}-\lambda_{n} \tau_{n}\left\|u_{n}-A w_{n}\right\|^{2}+2 \gamma_{n} \tau_{n} \lambda_{n} f_{2}\left(u_{n}, A x^{*}\right) \\
& +2 \alpha_{n} \tau_{n} f_{1}\left(z_{n}, x^{*}\right)-\tau_{n}\left\|z_{n}-h_{n}\right\|^{2}-\frac{1-\tau_{n}}{\tau_{n}}\left\|x_{n+1}-x_{n}\right\|^{2}+\left(1+\lambda_{n}\right) \delta_{n} \\
= & \left(1+\eta_{n}\right)\left\|x_{n}-x^{*}\right\|^{2}-\eta_{n}\left\|x_{n-1}-x^{*}\right\|^{2}+\xi_{n}\left\|x_{n}-x_{n-1}\right\|^{2} \\
& -\tau_{n} \lambda_{n}(1-\mu \kappa)\left\|y_{n}-A w_{n}\right\|^{2}-\lambda_{n} \tau_{n}\left\|u_{n}-A w_{n}\right\|^{2}+2 \gamma_{n} \tau_{n} \lambda_{n} f_{2}\left(u_{n}, A x^{*}\right) \\
& +2 \alpha_{n} \tau_{n} f_{1}\left(z_{n}, x^{*}\right)-\tau_{n}\left\|z_{n}-h_{n}\right\|^{2}-\frac{1-\tau_{n}}{\tau_{n}}\left\|x_{n+1}-x_{n}\right\|^{2}+\left(1+\lambda_{n}\right) \delta_{n},
\end{aligned}
$$

where

$$
\delta_{n}:=\frac{2 \beta_{n} \varepsilon_{n}}{\rho_{n}}+\beta_{n}^{2}, \eta_{n}:=\tau_{n} \theta_{n}, \xi_{n}:=\tau_{n} \theta_{n}\left(1+\theta_{n}\right)
$$

Set

$$
\Gamma_{n}:=\left\|x_{n}-x^{*}\right\|^{2}-\eta_{n}\left\|x_{n}-x^{*}\right\|^{2}+\xi_{n}\left\|x_{n}-x_{n-1}\right\|^{2} .
$$

Since $\left\{\tau_{n}\right\}$ and $\left\{\theta_{n}\right\}$ are non-decreasing sequences, we have that $\left\{\eta_{n}\right\}$ is a non-decreasing sequence. Hence

$$
\begin{aligned}
\Gamma_{n+1}-\Gamma_{n}= & \left\|x_{n+1}-x^{*}\right\|^{2}-\left(1+\eta_{n+1}\right)\left\|x_{n}-x^{*}\right\|^{2}+\eta_{n}\left\|x_{n-1}-x^{*}\right\|^{2} \\
& +\xi_{n+1}|| x_{n+1}-x_{n}\left\|^{2}-\xi_{n}|| x_{n}-x_{n-1}\right\|^{2} . \\
\leq & \left\|x_{n+1}-x^{*}\right\|^{2}-\left(1+\eta_{n}\right)\left\|x_{n}-x^{*}\right\|^{2}+\eta_{n}\left\|x_{n-1}-x^{*}\right\|^{2} \\
& +\xi_{n+1}|| x_{n+1}-x_{n}\left\|^{2}-\xi_{n}|| x_{n}-x_{n-1}\right\|^{2} .
\end{aligned}
$$

It then follows from (3.22) and (3.23) that

$$
\begin{aligned}
\Gamma_{n+1}-\Gamma_{n} \leq & -\tau_{n} \lambda_{n}(1-\mu \kappa)\left\|y_{n}-A w_{n}\right\|^{2}-\tau_{n} \lambda_{n}\left\|u_{n}-A w_{n}\right\|^{2}+2 \gamma_{n} \tau_{n} \lambda_{n} f_{2}\left(u_{n}, A x^{*}\right) \\
& +2 \alpha_{n} \tau_{n} f_{1}\left(z_{n}, x^{*}\right)-\tau_{n}\left\|z_{n}-h_{n}\right\|^{2}+\left(1+\lambda_{n}\right) \delta_{n} \\
& +\xi_{n+1}\left\|x_{n+1}-x_{n}\right\|^{2}-\frac{1-\tau_{n}}{\tau_{n}}\left\|x_{n+1}-x_{n}\right\|^{2}
\end{aligned}
$$

Now, since $x^{*} \in E P\left(f_{1}, C\right)$ and $z_{n} \in C$, we have $f_{1}\left(x^{*}, z_{n}\right) \geq 0$. Thus, by the pseudo-monotonicity of $f_{1}$, we have $f_{1}\left(z_{n}, x^{*}\right) \leq 0$. Similarly, since $A x^{*} \in E P\left(f_{2}, Q\right)$, we also have $f_{2}\left(u_{n}, A x^{*}\right) \leq 0$. 
Moreover, from [36], we have

$$
\begin{aligned}
\frac{1-\tau_{n}}{\tau_{n}}-\xi_{n+1} & =\frac{1}{\tau_{n}}-1-\tau_{n+1} \theta_{n+1}\left(1+\theta_{n+1}\right) \\
& \geq 2+\sigma-1-\frac{2}{2+\sigma} \\
& =\sigma+\frac{\sigma}{2+\sigma} \geq \sigma .
\end{aligned}
$$

Therefore, from (3.23), we have

$$
\begin{aligned}
\Gamma_{n+1}-\Gamma_{n} & \leq-\left(\frac{1-\alpha_{n}}{\alpha_{n}}-\xi_{n+1}\right)\left\|x_{n+1}-x_{n}\right\|^{2}+\left(1+\lambda_{n}\right) \delta_{n} \\
& \leq-\sigma\left\|x_{n+1}-x_{n}\right\|^{2}+\left(1+\lambda_{n}\right) \delta_{n}
\end{aligned}
$$

which yields

$$
\Gamma_{n+1} \leq \Gamma_{n}+\left(1+\lambda_{n}\right) \delta_{n}
$$

Using Lemma 2.4 and the fact that $\sum_{n=1}^{\infty}\left(1+\lambda_{n}\right) \delta_{n}<\infty$, we conclude that the sequence $\left\{\Gamma_{n}\right\}$ converges and consequently, $\left\{\Gamma_{n}\right\}$ is bounded. Therefore, there exists $\bar{\Gamma}>0$ such that $\Gamma_{n} \leq$ $\bar{\Gamma} \forall n \geq 0$. Clearly, from (3.27), we have

$$
\Gamma_{n} \leq \Gamma_{1}+\sum_{i=1}^{n-1}\left(1+\lambda_{i}\right) \delta_{i}
$$

Furthermore,

$$
\begin{aligned}
\Gamma_{n} & =\left\|x_{n}-x^{*}\right\|^{2}-\eta_{n}\left\|x_{n-1}-x^{*}\right\|^{2}+\xi_{n}\left\|x_{n}-x_{n-1}\right\|^{2} \\
& \geq\left\|x_{n}-x^{*}\right\|^{2}-\eta_{n}\left\|x_{n-1}-x^{*}\right\|^{2}
\end{aligned}
$$

and

$$
\eta_{n}=\tau_{n} \theta_{n} \leq \frac{1}{2+\sigma}=: \eta<1 .
$$

It then follows from (3.29) and (3.30) that

$$
\begin{aligned}
\left\|x_{n}-x^{*}\right\|^{2} & \leq \eta_{n}\left\|x_{n-1}-x^{*}\right\|^{2}+\Gamma_{n} \\
& \leq \eta\left\|x_{n-1}-x^{*}\right\|^{2}+\bar{\Gamma} \\
& \vdots \\
& \leq \eta^{n}\left\|x_{0}-x^{*}\right\|^{2}+\bar{\Gamma}\left(\eta^{n-1}+\cdots+1\right) \\
& \leq \eta^{n}\left\|x_{0}-x^{*}\right\|^{2}+\frac{\bar{\Gamma}}{1-\eta} .
\end{aligned}
$$

Again,

$$
\begin{aligned}
\Gamma_{n+1} & =\left\|x_{n+1}-x^{*}\right\|^{2}-\eta_{n+1}\left\|x_{n}-x^{*}\right\|^{2}+\xi_{n+1}\left\|x_{n+1}-x_{n}\right\|^{2} \\
& \geq-\eta_{n+1}\left\|x_{n}-x^{*}\right\|^{2} .
\end{aligned}
$$


From (3.31) and (3.32), we obtain

$$
\begin{aligned}
-\Gamma_{n+1} & \leq \eta_{n+1}\left\|x_{n}-x^{*}\right\|^{2} \\
& \leq \eta\left\|x_{n}-x^{*}\right\|^{2} \\
& \leq \eta^{n+1}\left\|x_{0}-x^{*}\right\|^{2}+\frac{\eta \bar{\Gamma}}{1-\eta} .
\end{aligned}
$$

It follows from (3.26) and (3.33) that

$$
\begin{aligned}
\sigma \sum_{n=1}^{k}\left\|x_{n+1}-x_{n}\right\|^{2} & \leq \Gamma_{1}-\Gamma_{n+1}+\sum_{n=1}^{k}\left(1+\lambda_{n}\right) \delta_{n} \\
& \leq \bar{\Gamma}-\Gamma_{k+1}+\sum_{n=1}^{k}\left(1+\lambda_{n}\right) \delta_{n} \\
& \leq \eta^{k+1}\left\|x_{0}-x^{*}\right\|^{2}+\frac{\bar{\Gamma}}{1-\eta}+\sum_{n=1}^{k}\left(1+\lambda_{n}\right) \delta_{n} \\
& \leq\left\|x_{0}-x^{*}\right\|^{2}+\frac{\bar{\Gamma}}{1-\eta}+\sum_{n=1}^{k}\left(1+\lambda_{n}\right) \delta_{n},
\end{aligned}
$$

which implies that

$$
\sum_{n=1}^{\infty}\left\|x_{n+1}-x_{n}\right\|^{2}<\infty
$$

and

$$
\lim _{n \rightarrow \infty}|| x_{n+1}-x_{n} \|=0
$$

From (3.22), we have

$$
\begin{aligned}
\left\|x_{n+1}-x^{*}\right\|^{2} \leq & \left(1+\eta_{n}\right)\left\|x_{n}-x^{*}\right\|^{2}-\eta_{n}\left\|x_{n-1}-x^{*}\right\| \\
& +2\left\|x_{n-1}-x_{n}\right\|^{2}+\left(1+\lambda_{n}\right) \delta_{n} .
\end{aligned}
$$

Thus, by (3.35), (3.37) and Lemma 2.3, we have that there exists $l \in[0, \infty)$ such that

$$
\lim _{n \rightarrow \infty}\left\|x_{n}-x^{*}\right\|^{2}=l \text {. }
$$

Again, from (3.21), we get

$$
\lim _{n \rightarrow \infty}\left\|w_{n}-x^{*}\right\|^{2}=l
$$

From the definition of $w_{n}$, we have

$$
\lim _{n \rightarrow \infty}\left\|w_{n}-x_{n}\right\|=\lim _{n \rightarrow \infty} \theta_{n}|| x_{n}-x_{n-1}\left\|\leq \lim _{n \rightarrow \infty}|| x_{n}-x_{n-1}\right\|=0 .
$$

Observe that

$$
\left\|x_{n+1}-\left.x^{*}\right|^{2} \leq\left(1-\tau_{n}\right)\right\| x_{n}-\left.x^{*}\right|^{2}+\tau_{n}\left\|h_{n}-x^{*}\right\|^{2} .
$$

This implies that

$$
\left\|h_{n}-x^{*}\right\|^{2} \geq \frac{\left\|x_{n+1}-\left.x^{*}\right|^{2}-\right\| x_{n}-\left.x^{*}\right|^{2}}{\tau_{n}}+\left\|x_{n}-x^{*}\right\|^{2} .
$$


Since $\left\{\tau_{n}\right\}$ is bounded, it follows from (3.38) and (3.42) that

$$
\lim _{n \rightarrow \infty}\left\|h_{n}-x^{*}\right\| \geq \lim _{n \rightarrow \infty}\left\|x_{n}-x^{*}\right\|^{2}=l \text {. }
$$

On the other hand, from (3.9) and (3.19), we have

$$
\lim _{n \rightarrow \infty}\left\|h_{n}-x^{*}\right\|^{2} \leq \lim _{n \rightarrow \infty}\left\|z_{n}-x^{*}\right\|^{2} \leq \lim _{n \rightarrow \infty}\left\|w_{n}-x^{*}\right\|^{2}=l .
$$

Combining (3.43) and (3.44), we have that

$$
\begin{gathered}
\lim _{n \rightarrow \infty}\left\|h_{n}-x^{*}\right\|^{2}=l . \\
\left\|x_{n}-h_{n}\right\|=\frac{1}{\tau_{n}}\left\|x_{n+1}-x_{n}\right\|^{2} \rightarrow 0, n \rightarrow \infty .
\end{gathered}
$$

Also, from (3.22), we obtain

$$
\begin{gathered}
\tau_{n} \lambda_{n}(1-\mu \kappa)\left\|y_{n}-A w_{n}\right\|^{2}+\tau_{n} \lambda_{n}\left\|u_{n}-A w_{n}\right\|^{2} \\
-2 \gamma_{n} \tau_{n} \lambda_{n} f_{2}\left(u_{n}, A x^{*}\right)+\tau_{n}\left\|z_{n}-h_{n}\right\|^{2}-2 \alpha_{n} \tau_{n} f_{1}\left(z_{n}, x^{*}\right) \\
\leq\left(1+\eta_{n}\right)\left\|x_{n}-x^{*}\right\|^{2}-\eta_{n}\left\|x_{n-1}-x^{*}\right\|^{2} \\
-\left\|x_{n+1}-x^{*}\right\|^{2}+\left(1+\lambda_{n}\right) \delta_{n} \rightarrow 0, n \rightarrow \infty
\end{gathered}
$$

Therefore,

$$
\lim _{n \rightarrow \infty}\left\|y_{n}-A w_{n}\right\|=\lim _{n \rightarrow \infty}\left\|u_{n}-A w_{n}\right\|=\lim _{n \rightarrow \infty}\left\|z_{n}-h_{n}\right\|=0
$$

and

$$
\lim _{n \rightarrow \infty} f_{2}\left(u_{n}, A x^{*}\right)=\lim _{n \rightarrow \infty} f_{1}\left(z_{n}, x^{*}\right)=0
$$

Consequently,

$$
\begin{gathered}
\left\|x_{n}-z_{n}\right\| \leq\left\|x_{n}-h_{n}\right\|+\left\|z_{n}-h_{n}\right\| \rightarrow 0, n \rightarrow \infty, \\
\left\|y_{n}-u_{n}\right\| \leq\left\|y_{n}-A w_{n}\right\|+\left\|u_{n}-A w_{n}\right\| \rightarrow 0, n \rightarrow \infty,
\end{gathered}
$$

and

$$
\left\|w_{n}-h_{n}\right\| \leq\left\|w_{n}-x_{n}\right\|+\left\|x_{n}-h_{n}\right\| \rightarrow 0, n \rightarrow \infty .
$$

Since $\left\{z_{n}\right\}$ is bounded, there exists a subsequence $\left\{z_{n_{k}}\right\}$ of $\left\{z_{n}\right\}$ converging weakly to $x^{+} \in C$. Therefore, it follows from the upper semicontinuity of $f_{1}\left(\cdot, x^{*}\right)$ that

$$
f_{1}\left(x^{+}, x^{*}\right) \geq \limsup _{k \rightarrow \infty} f_{1}\left(z_{n_{k}}, x^{*}\right)=\lim _{k \rightarrow \infty} f_{1}\left(z_{n_{k}}, x^{*}\right)=\lim _{n \rightarrow \infty} f_{1}\left(z_{n}, x^{*}\right)=0 .
$$

Moreover, since $x^{*} \in E P\left(f_{1}, C\right)$ and $x^{+} \in C$, we have $f_{1}\left(x^{*}, x^{+}\right) \geq 0$. Hence, by the pseudomonotonicity of $f_{1}$, we get that $f_{1}\left(x^{+}, x^{*}\right) \leq 0$. This with (3.48) implies that $f_{1}\left(x^{+}, x^{*}\right)=0$. Using condition $A 4$, we conclude that $x^{+} \in E P\left(f_{1}, C\right)$. Since $\left\{z_{n_{k}}\right\}$ converges weakly to $x^{+}$, we have that $\left\{w_{n_{k}}\right\}$ converges weakly to $x^{+}$. Therefore, since $A$ is continuous, we have that $\left\{A w_{n_{k}}\right\}$ converges weakly to $A x^{+}$. From $\lim _{n \rightarrow \infty}|| u_{n}-A w_{n} \|=0$, we have that $u_{n_{k}}$ converges weakly to $A x^{+}$. Hence by the same line of argument above, we conclude that $A x^{+} \in E P\left(f_{2}, Q\right)$ immediately.

Theorem 3.2. Let $f_{1}$ be strongly pseudomonotone on $C$, i.e., $f_{1}(x, y) \geq 0 \Rightarrow f_{1}(y, x) \leq-\gamma \| x-$ $y \|^{2}, \forall x, y \in C$ for some $\gamma>0$. Suppose that $2 \alpha_{n} \gamma>1, \sum_{n=0}^{\infty} \tau_{n}=\infty$, and $\Omega \neq \emptyset$. Then, the sequence $\left\{x_{n}\right\}$ generated by Algorithm 3.1 converges strongly to some $x^{+} \in \Omega$. 
Proof. By similar steps as in the proof of Theorem 3.1 and the fact that the strong pseudomonotonicity implies the pseudomonotonicity, we have that $\left\{x_{n}\right\}$ converges weakly to some $x^{+} \in \Omega$. From (3.11), we have

$$
\begin{aligned}
\left\|x_{n+1}-x^{+}\right\|^{2} \leq & \left(1-\tau_{n}\right)\left\|x_{n}-x^{+}\right\|^{2}+\tau_{n}\left\|z_{n}-x^{+}\right\|^{2}+2 \alpha_{n} \tau_{n} f_{1}\left(z_{n}, x^{+}\right) \\
& -\tau_{n}\left\|z_{n}-h_{n}\right\|^{2}+\frac{2 \tau_{n} \beta_{n} \varepsilon_{n}}{\rho_{n}}+2 \beta_{n}^{2} \tau_{n}-\tau_{n}\left(1-\tau_{n}\right)\left\|x_{n}-h_{n}\right\|^{2} \\
\leq & \left(1-\tau_{n}\right)\left\|x_{n}-x^{+}\right\|^{2}+\tau_{n}\left\|z_{n}-x^{+}\right\|^{2}+2 \alpha_{n} \tau_{n} f_{1}\left(z_{n}, x^{+}\right) \\
& +\frac{2 \tau_{n} \beta_{n} \varepsilon_{n}}{\rho_{n}}+2 \beta_{n}^{2} \tau_{n} .
\end{aligned}
$$

Since $x^{+} \in E P\left(f_{1}, C\right)$ and $z_{n} \in C$, we have that $f_{1}\left(x^{+}, z_{n}\right) \geq 0$. Since $f_{1}$ is strongly pseudomonotone, we have that $f_{1}\left(z_{n}, x^{+}\right) \leq-\gamma\left\|z_{n}-x^{+}\right\|^{2}$. It follows from $2 \alpha_{n} \gamma>1$ and (3.49) that

$$
\begin{aligned}
\left\|x_{n+1}-x^{+}\right\|^{2} \leq & \left(1-\tau_{n}\right)\left\|x_{n}-x^{+}\right\|^{2}+\tau_{n}\left\|z_{n}-x^{+}\right\|^{2}-2 \alpha_{n} \tau_{n} \gamma\left\|z_{n}-x^{+}\right\|^{2} \\
& +\frac{2 \tau_{n} \beta_{n} \varepsilon_{n}}{\rho_{n}}+2 \beta_{n}^{2} \tau_{n} \\
\leq & \left(1-\tau_{n}\right)\left\|x_{n}-x^{+}\right\|^{2}+\frac{2 \tau_{n} \beta_{n} \varepsilon_{n}}{\rho_{n}}+2 \beta_{n}^{2} \tau_{n} .
\end{aligned}
$$

Applying Lemma 2.6 to (3.50) with $a_{n}=\left\|x_{n}-x^{+}\right\|^{2}, \gamma_{n}=\tau_{n}$ and $\delta_{n}=\frac{2 \beta_{n} \varepsilon_{n}}{\rho_{n}}+2 \beta_{n}^{2}$, we conclude that $\left\{x_{n}\right\}$ converges strongly to $x^{+} \in \Omega$ easily.

\section{THE APPLICATIONS TO THE SPLIT OPTIMIZATION PROBLEMS}

Let $g_{1}: C \rightarrow \mathbb{R}$ and $g_{2}: Q \rightarrow \mathbb{R}$ be functions, where $C$ and $Q$ are nonempty closed and convex subsets of real Hilbert spaces $H_{1}$ and $H_{2}$, respectively. Let $A: H_{1} \rightarrow H_{2}$ be a bounded linear operator. The split optimization is to find $x^{*} \in C$ such that

$$
g_{1}\left(x^{*}\right) \leq g_{1}(x), \forall x \in C
$$

and such that $y^{*}=A x^{*}$ satisfies

$$
g_{2}\left(y^{*}\right) \leq g_{2}(y), \forall y \in Q .
$$

Let $\Omega_{1}$ be the solution set of the split optimization problem (4.1)-(4.2).

Lemma 4.1. [37, Proposition 4.1] Let $g: C \rightarrow \mathbb{R}$ be an arbitrary function. Define the bifunction $f(x, y):=g(y)-g(x), \forall x, y \in C$. Then

(i). The bifunction $f$ is paramonotone on $C$.

(ii). The solution set EP $(f, C)$ coincides with the solution set of the optimization problem

$$
\min \{g(x): x \in C\} .
$$

Therefore, if we let $f_{1}(x, y)=g_{1}(y)-g_{1}(x)$ and $f_{2}(x, y)=g_{2}(y)-g_{2}(x)$ and assume that $g_{1}$ and $g_{2}$ satisfy the following conditions on $C$ and $Q$, respectively

(B1). $g_{1}$ and $g_{2}$ are weakly lower continuous and $\varepsilon$-subdifferential functions, (B2). the mappings $x \mapsto \partial g_{1}(x)$ and $x \mapsto \partial g_{2}(x)$ are bounded on bounded subsets of $C$ and $Q$, Conditions $(B 1)$ and $(B 2)$ and Lemma 4.3 imply that $f_{1}$ and $f_{2}$ satisfy conditions $(A 1)-(A 4)$.

Now, we show how Algorithm (3.1) can be adapted to solve split optimization problem (4.1)(4.2). 
Algorithm 4.1. For initial $x_{0}, x_{1} \in C, \lambda_{1}>0, \mu \in(0,1)$ and non-negative parameter sequences $\left\{\rho_{n}\right\},\left\{\beta_{n}\right\},\left\{\tau_{n}\right\},\left\{\theta_{n}\right\}$ and $\left\{\varepsilon_{n}\right\}$ satisfying the following conditions,

1. $\rho_{n} \geq \rho>0, \beta_{n}>0, \varepsilon_{n}>0$,

2. $\sum_{n=1}^{\infty} \frac{\beta_{n} \varepsilon_{n}}{\rho_{n}}<+\infty, \sum_{n=1}^{\infty} \beta_{n}^{2}<+\infty$,

3. $\left\{\tau_{n}\right\}$ and $\left\{\theta_{n}\right\}$ are nondecreasing sequences,

4. $0 \leq \theta_{n} \leq 1, \tau<\tau_{n} \leq \frac{1}{2+\sigma}, \tau>0, \sigma>0$,

calculate $x_{n+1}$ as follows:

Step 1. Set $w_{n}=x_{n}+\theta_{n}\left(x_{n}-x_{n-1}\right)$, select $v_{n} \in \partial_{\varepsilon_{n}} g_{2}\left(u_{n}\right)$, where $u_{n}=P_{Q}\left(A w_{n}\right)$, and compute $\gamma_{n}=\frac{\beta_{n}}{\max \left\{\rho_{n},\left\|v_{n}\right\|\right\}}, y_{n}=P_{Q_{n}}\left(u_{n}-\gamma_{n} v_{n}\right)$, where $Q_{n}$ is the set defined by

$$
Q_{n}=\left\{y \in H_{2}:\left\langle y-u_{n}, A w_{n}-u_{n}\right\rangle \leq 0\right\} .
$$

Step 2. Compute $z_{n}=P_{C}\left(w_{n}+\lambda_{n} A^{*}\left(y_{n}-A w_{n}\right)\right)$ and

$$
\lambda_{n+1}=\left\{\begin{array}{l}
\min \left\{\frac{\mu\left\|y_{n}-A w_{n}\right\|^{2}}{\left\|A^{*}\left(y_{n}-A w_{n}\right)\right\|^{2}}, \lambda_{n}\right\}, \text { if }\left\|A^{*}\left(y_{n}-A w_{n}\right)\right\| \neq 0, \\
\lambda_{n}, \\
\text { Otherwise. }
\end{array}\right.
$$

Step 3. Select $q_{n} \in \partial_{\varepsilon_{n}} g_{1}\left(z_{n}\right)$ and compute $\alpha_{n}=\frac{\beta_{n}}{\max \left\{\rho_{n},\left\|q_{n}\right\|\right\}}, x_{n+1}=\left(1-\tau_{n}\right) x_{n}+\tau_{n} P_{C_{n}}\left(z_{n}-\right.$ $\left.\alpha_{n} q_{n}\right)$. The set $C_{n}$ is defined by

$$
C_{n}=\left\{x \in H_{1}:\left\langle x-z_{n}, w_{n}+\lambda_{n} A^{*}\left(y_{n}-A w_{n}\right)-z_{n}\right\rangle \leq 0\right\} .
$$

Corollary 4.1. Let $\left\{x_{n}\right\}$ be the sequence generated by Algorithm 4.1 and suppose that $\Omega_{1} \neq \emptyset$. Then $\left\{x_{n}\right\}$ converges weakly to some $x^{+} \in \Omega_{1}$.

\section{CONCLUSIONS}

In this paper, a new inertial-type iterative algorithm, which does not require any knowledge of the operator norm, was presented for solving the split equilibrium problem with bifunctions. The new algorithm was devised without computing proximal or resolvent operators. The step size is our algorithm is self-adaptive and we also added the inertial extrapolation term to speed up the convergence of the iterative algorithm. A weak convergence theorem was proved under standard conditions imposed on the cost functions and with the extra condition that $f_{1}$ is strongly pseudomonotone. We also obtained a strong convergence result.

Acknowledgments The author acknowledges with thanks to referees for the useful suggestions which are helpful for the improvement of the presentation of this paper.

\section{REFERENCES}

[1] L.V. Nguyen, Q.H. Ansari, X. Qin, Linear conditioning, weak sharpness and finite convergence for equilibrium problems, J. Glob. Optim. 77 (2020), 405-424.

[2] F. Alvarez, H. Attouch, An inertial proximal method for maximal monotone operators via discretization of a nonlinear oscillator with damping, Set-Valued Anal. 9 (2001), 3-11.

[3] H. Attouch, X. Goudon, P. Redont, The heavy ball with friction. I. The continuous dynamical system, Commun. Contemp. Math. 2 (2000), 1-34.

[4] H. Attouch, M.O. Czarnecki, Asymptotic control and stabilization of nonlinear oscillators with nonisolated equilibria, J. Differential Equations 179 (2002), 278-310. 
[5] X. Qin, N.T. An, Smoothing algorithms for computing the projection onto a Minkowski sum of convex sets, Comput. Optim. Appl. 74 (2019), 821-850.

[6] X. Qin, Y.J. Cho, S.M. Kang, Convergence theorems of common elements for equilibrium problems and fixed point problems in Banach spaces, J. Comput. Appl. Math. 225 (2009), 20-30.

[7] F.U. Ogbuisi, Popov subgradient extragradient algorithm for pseudomonotone equilibrium problems in Banach spaces, J. Nonlinear Funct. Anal. 2019 (2019), Article ID 44.

[8] F.U. Ogbuisi, Y. Shehu, A projected subgradient-proximal method for split equality equilibrium problems of pseudomonotone bifunctions in Banach spaces, J. Nonlinear Var. Anal. 3 (2019), 205-224.

[9] Y. Shehu, F.U. Ogbuisi, Approximation of a common fixed point of left Bregman strongly nonexpansive mappings and solution of equilibrium problems, J. Appl. Anal. 21 (2015), 63-78.

[10] M.O. Osilike, E.E. Chima, Mixed equilibrium and multiple-set split feasibility problems for asymptotically k-strictly pseudononspreading mappings, Commun. Optim. Theory, 2020 (2020), Article ID 14.

[11] E. Blum, W. Oettli, From optimization and variational inequalities to equilibrium problems, Math. Stud. 63 (1994), 123-145.

[12] X. Qin, J.C. Yao, A viscosity iterative method for a split feasibility problem, J. Nonlinear Convex Anal. 20 (2019), 1497-1506.

[13] C.E. Chidume, A. Adamu, On split generalized mixed equality equilibrium and split equality fixed point problems, Appl. Set-Valued Anal. Optim. 2 (2020), 273-283.

[14] K.R. Kazmi, S.H. Rizvi, Iterative approximation of a common solution of a split equilibrium problem, a variational inequality problem and a fixed point problem, J. Egyptian Math. Soc. 21 (2013), 44-51.

[15] A. Moudafi, Split monotone variational inclusions, J. Optim. Theory Appl. 150 (2011), 275-283.

[16] P.L. Combettes, S.A. Hirstoaga, Equilibrium programming in Hilbert spaces, J. Nonlinear Convex Anal. 6 (2005), 117-136.

[17] F.U. Ogbuisi, O.T. Mewomo, On split generalised mixed equilibrium problems and fixed-point problems with no prior knowledge of operator norm, J. Fixed Point Theory Appl. 19 (2017), 2109-2128.

[18] D.V. Hieu, Parallel extragradient-proximal methods for split equilibrium problems, Math. Model. Anal. 21 (2016), 478-501.

[19] S.Y. Cho, A monotone Bregan projection algorithm for fixed point and equilibrium problems in a reflexive Banach space, Filomat, 34 (2020), 1487-1497.

[20] D.V. Hieu, Halpern subgradient extragradient method extended to equilibrium problems, Rev. R. Acad. Cienc. Exactas Fis. Nat. Ser. A Mat. RACSAM 111 (2017), 823-840.

[21] S.Y. Cho, X. Qin, On the strong convergence of an iterative process for asymptotically strict pseudocontractions and equilibrium problems, Appl. Math. Comput. 235 (2014), 430-438.

[22] D.V. Hieu, L.D. Muu, P.K. Anh, Parallel hybrid extragradient methods for pseudomonotone equilibrium problems and nonexpansive mappings, Numer. Algo. 73 (2016), 197-217.

[23] A. Moudafi, Proximal point algorithm extended to equilibrum problems, J. Nat. Geom. 15 (1999), 91-100.

[24] D.V. Hieu, Projection method for solving split equilibrium problems, J. Ind. Manag. Optim. 16 (2020), 23312349.

[25] F. Alvarez, Weak convergence of a relaxed and inertial hybrid projection-proximal point algorithm for maximal monotone operators in Hilbert space, SIAM J. Optim. 14 (2003), 773-782.

[26] H. Attouch, J. Peypouquet, P. Redont, A dynamical approach to an inertial forward-backward algorithm for convex minimization, SIAM J. Optim. 24 (2014), 232-256.

[27] D.A. Lorenz, T. Pock, An inertial forward-backward algorithm for monotone inclusions, J. Math. Imaging Vision 51 (2015), 311-325.

[28] X. Qin, L. Wang, J.C. Yao, Inertial splitting method for maximal monotone mappings, J. Nonlinear Convex Anal. 21 (2020), 2325-2333.

[29] R.I. Bot, E.R. Csetnek, C. Hendrich, Inertial Douglas-Rachford splitting for monotone inclusion, Appl. Math. Comput. 256 (2015), 472-487.

[30] C. Chen, R.H. Chan, S. Ma, J. Yang, Inertial proximal ADMM for linearly constrained separable convex optimization, SIAM J. Imaging Sci. 8 (2015), 2239-2267.

[31] R.I. Bot, E.R. Csetnek, An inertial forward-backward-forward primal-dual splitting algorithm for solving monotone inclusion problems, Numer. Algor. 71 (2016), 519-540. 
[32] M. Tian, M. Tong, Self-adaptive subgradient extragradient method with inertial modification for solving monotone variational inequality problems and quasi-nonexpansive fixed point problems, J. Inequal. Appl. 2019 (2019), Article ID 7.

[33] L.V. Long, D.V. Thong, V.T. Dung, New algorithms for the split variational inclusion problems and application to split feasibility problems, Optimization 68 (2019), 2335-2363.

[34] S.S. Chang, Some problems and results in the study of nonlinear analysis, Nonlinear Anal. 30 (1997), 41974208.

[35] H.K. Xu, Iterative algorithms for nonlinear operators, J. London. Math. Soc. 2 (2002), 240-256.

[36] K. Sitthithakerngkiet, J. Deepho, P. Kumam, A hybrid viscosity algorithm via modify the hybrid steepest descent method for solving the split variational inclusion in image reconstruction and fixed point problems, Appl Math Comput. 250 (2015), 986-1001.

[37] P.K. Anh, T.N. Hai, Novel self-adaptive algorithms for non-Lipschitz equilibrium problems with applications, J. Glob. Optim. 73 (2019), 637-657. 